\title{
Topological study of the convergence in the voter model
}

\author{
Inés Caridi ${ }^{1 *}$ (D), Sergio Manterola2 ${ }^{2}$, Viktoriya Semeshenko ${ }^{3,4}$ and Pablo Balenzuela ${ }^{2,5}$
}

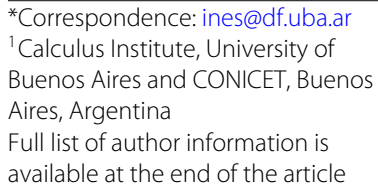

*Correspondence: ines@df.uba.ar ${ }^{1}$ Calculus Institute, University of Buenos Aires and CONICET, Buenos Aires, Argentina

Full list of author information is available at the end of the article

\begin{abstract}
The voter model has been widely studied due to its simple formulation and attainable theoretical treatment. The study of the "active links", edges that connect nodes in different states, has been a key element in the analysis of the convergence of the model. Typically, the density of active links, $\rho$, is used to characterize the system when approaching to absorbing state. However, more information can be extracted from how the active links are distributed across the underlying network.

In this paper we study the dynamics of active links in the voter model, from a perspective of complex networks. This approach allows us to understand how the dynamics of the model is mapped in topological features of a dynamical network of active links. We found that certain topological properties of the Active Link Network show salient features related to the dynamics of the model. The Active Link Network goes from a state similar to the underlying random network in the initial state to extremely disassortative graph when the dynamics approaches to absorbing state. In this state, the active link network is dominated by "star-like" motifs, where different opinions take different topological roles on the network. Thus, the Active Link Network shows some properties which are distant from the hypothesis made by the current theoretical models, which assume there are no correlations among active links.
\end{abstract}

\section{Introduction}

The voter model is one of the most well-known models with non-equilibrium dynamics. It has been studied extensively as a paradigmatic opinion dynamics model in the framework of probability theory (Clifford and Sudbury 1973; Liggett 1999) and statistical physics, and is one of the few non-equilibrium stochastic processes that can be solved exactly (Redner 2001). The model consists of $N$ agents placed in the nodes of an interacting network. Each agent is characterized by a single variable $s$ which can take values +1 or -1 . The standard rules of the model establish that at each time step $t$, a random individual is selected (agent $i$ ) and a random neighbour is selected among his nearest neighbours (agent $j$ ). If the states of agents are different, agent $i$ imitates the state of agent $j$, otherwise the states remain unchanged. This imitation dynamics has been proposed as a social influence mechanism in contexts of great uncertainty (Bikhchandani et al. 1992).

Starting from random initial conditions, these simple rules tend to increase the order of the system. For instance, in finite systems, this dynamics gives place to the emergence of the consensus of one of the two states. The time to reach consensus depends on the size $N$ and dimension $d$ of the system (Cox 1989). An interesting question that appears is whether full consensus is reached in a system of infinite size.

(c) The Author(s). 2019 Open Access This article is distributed under the terms of the Creative Commons Attribution 4.0 International License (http://creativecommons.org/licenses/by/4.0/), which permits unrestricted use, distribution, and reproduction in any medium, provided you give appropriate credit to the original author(s) and the source, provide a link to the Creative Commons license, and indicate if changes were made. 
The voter model has been solved exactly for hypercubic lattices of arbitrary dimensions using different approaches. Early studies performed by probabilists (Clifford and Sudbury 1973; Holley and Liggett 1975; Liggett 1985; Cox and Griffeath 1986) used the fact that the model can be exactly mapped on a model of random walkers that coalesce upon encounter. Another approach based on master equations in order to derive the general solution on lattices was followed in (Krapivsky 1992; Frachebourg and Krapivsky 1996). The authors used the master equation for the probability distribution function for the state $S$ of the system to derive the asymptotic behavior of the density of active links (or interface), and concluded that the voter model reaches consensus for dimensions $d \leq 2$. For $d>2$, instead, it exhibits asymptotically a finite density of links which means that no consensus is reached. The density of active links is a useful measure to describe the dynamics of the system towards its asymptotic state and it is a good quantity to use as an order parameter, given that it is nonzero while the system is not in one of the absorbing states and zero otherwise.

The original voter model does not include the possibility of spontaneous changes in the variable $s$ given that the state of a node only changes through interaction with nearest neighbors. However, the noisy voter model (Scheucher and Spohn 1988; Granovsky and Madras 1995; de Oliveira et al. 1993) incorporates this option producing the disappearance of long range order (de Oliveira et al. 1993).

Given its simplicity, the voter model has been studied under several modifications. For instance, (Mobilia 2003; Mobilia et al. 2005; Mobilia et al. 2007) incorporate the presence of "zealots", i.e., individuals that does not change the opinions during dynamics. On the other hand, in (Vázquez et al. 2003; Vázquez and Redner 2004) the constrained voter model is analyzed. In this work, agents can be in three states (leftists, rightists, or centrists) but interactions involve only centrists. When the change of the state of the agent requires the agree of several of his neighbors, it is called the q-voter model and has been analyzed in Timpanaro and Galam (2014); Javarone and Squartini (2015). Several other modifications can be found in 67 (Castallano et al. 2009).

The collective dynamics can be modified if the underlying topology is not regular and is represented by complex networks (Castellano et al. 2003; Sood and Redner 2005; Suchecki et al. 2005a; Castellano 2005; Sood et al. 2008). In these cases, different ways of defining the voter dynamics, which are perfectly equivalent on regular lattices, give rise to nonequivalent generalizations of the voter model. The most natural generalization is called the direct voter model and consists in picking up a node and making it equal to one of its neighbors. With this rule the global magnetization is not longer conserved (Castallano et al. 2009; Suchecki et al. 2005b). The magnetization is conserved if a link updating rule is implemented (Suchecki et al. 2005b). If a selected node copies its state to a randomly selected neighbor it is called reverse voter model.

The voter model has been studied on different complex networks (Castellano et al. 2003; Sood and Redner 2005; Suchecki et al. 2005a; Castellano 2005; Sood et al. 2008), where various variables have been analyzed: mean consensus time in finite networks, the influence or degree of randomness, among others. The co-evolving voter model, in which nodes follow rules of the voter model and reorganization of the links takes place through rewiring dynamics, has been analyzed in (Holme and Newman 2006; Vazquez et al. 2008; Kimura and Hayakawa 2008; Durrett and et al. 2012; Böhme and Gross 2011; Demirel et al. 2014). The co-evolving voter model exhibits a fragmentation transition from a 
single connected network to a network with two components (Holme and Newman 2006; Vazquez et al. 2008).

From previous works we have seen that for an ensemble of finite complex networks, the density of active links $(\rho)$ follows an exponential decay from $\rho \simeq 0.5$ for a random initial state to zero as the dynamics converges to absorbing state (Suchecki et al. 2005a). However, the study of the active links in terms of networks has not been done yet.

The master equation for $\rho(t)$ was also used to analyze the dynamics towards absorbing state in complex uncorrelated networks where it was derived using the homogeneous pair approximation (Vazquez and Eguiluz 2008). An improvement to this analysis could be found in (Pugliese and Castellano 2009) where a heterogeneous pair approximation is developed allowing a dependence of the density of active links with the node degree, $\rho(k)$, generalizing the previous studies.

The above mentioned works provide the solutions in the thermodynamic limit, and assume that active links are uncorrelated. However, as it was suggested in (Böhme and Gross 2011), this assumption is not correct. The authors studied the fragmentation transition (FT) in an adaptive voter model. In this work, the voter model dynamics is analyzed in the vicinity of the fragmentation zone, where the density of active links $(\rho)$ is low, and noticed that active links can not be treated as independent because "they all become inert at once if the focal node reverts to its original opinion" (active links form star-like motifs in this regime). This correlation underlies the observed inaccuracy in mean field approximations which are overcame by the authors using a motif-like dynamics of multiple active links.

These ideas have been further explored in (Demirel et al. 2014), where the authors highlighted the importance of the heterogeneity of active links given by star-like motifs, and developed a theoretical description of fragmentation dynamics in the neighborhood of the transition.

Given that the distribution and dynamics of active links are crucial in order to understand the evolution of the model and related topics, we analyze numerically the sub-graph of the underlying network formed by the active links. In particular, we study the convergence process of the voter model in some complex networks by analyzing the evolution and topological properties of the Active Links Network. We observe that the system evolves from an Active Link Network similar to the underlying graphs (when $\rho \simeq 0.5$ ) to a graph dominated by star-like motifs, when $\rho$ is close to zero. This little structures are highly dissasortative, and there exist a strong correlation between the topological role of nodes and the value of their opinions.

\section{The model}

We consider the evolution of a direct voter model of $N$ agents immersed in a contact network, $G$. Here, $G$ is an Erdos-Renyi (ER) random network with mean degree $<k>=12$ although results for ER with mean degree $<k>=6$ and a Barabasi-Albert type are shown in Appendix A. Let us call $s_{i}$ the opinion state of $i$-agent, which can take two possible values: 1 and -1 . Initially, the states of agents are set up randomly with the same probability for each one.

The active links are edges that connect two agents with different opinion state in a contact network. The set of active links, changing along the dynamics, is a subset of the set 
of links of $G$. The Active Links Network, called $G_{A L}$ is defined as the induced sub-graph of $G$ from the set of active links. Hence, $G_{A L}$ is included in $G$.

Here is important to make clear the difference between the underlying (contact) network, which remains static during the implemented voter model dynamics, and the Active Links Network, which is a dynamical structure reflecting the dynamical state of the whole system.

In what follows, we analyze the topological properties of the Active Links Network $G_{A L}$, resulting from a voter model dynamics, in order to extract the information of the convergence process encoded in this topology.

As we mentioned in the Introduction, given the exponentially monotonic decaying relationship between the average ensemble of density of active links $\rho(t)$ in complex networks (Suchecki et al. 2005a), we describe the convergence process in terms of $\rho$, which makes it easier to calculate and compare the results with related problems (Böhme and Gross 2011; Demirel et al. 2014).

\section{Results}

In order to proceed with the analysis, we select two particular instants of the dynamics which correspond to different values of the parameter $\rho$ : early times corresponding to large values of $\rho(\simeq 0.5)$ and times close to convergence which correspond to small values of $\rho(\simeq 0.0)$.

In order to describe the Active Links Network, $G_{A L}$, we begin with the analysis of the connectivity properties of the network, and the comparison between the connectivity properties of nodes in both networks (the contact one and $G_{A L}$ ): the degree $k$ of an agent on the contact network, the mean degree $<k k>$ of his neighbors on the contact network, the degree of an agent on $G_{A L}, k_{A L}$, and the mean degree of his neighbors on $G_{A L}$, < $k k>A L$.

In order to visualize the main differences in the $G_{A L}$ at the different stages of the dynamics, we plot snapshots of $G_{A L}$ as shown in Fig. 1a, c.

In right panels of Fig. 1b, $\mathrm{d}$ we plot the degree distribution of the corresponding graph $G_{A L}$. We can observe that for large values of $\rho$, the result looks similar to the underlying static ER network. It is the expected result given that the dynamics does not alter the collective state of the system. Empty points correspond to states -1 , and filled points to states 1.

However, if we look at the $G_{A L}$ close to the converged state, we can appreciate that the degree distribution is completely different from the previous one, and it is dominated by a high number of nodes with degree one and two. We can also observe a small peak of nodes with higher degree. This is consistent with the star-like motifs observed in the snapshots. We can see from the quasi converged snapshot, that the nodes in the dominant state are all with degree one (standing for the role of leaves of the stars), or degree two (connecting two stars), and eventually, degree three (connecting three stars).

In order to understand how collective properties of the model are mapped into the $G_{A L}$, we plot the degree of nodes in $G_{A L}\left(k_{A L}\right)$ versus the degree of nodes in the underlying static network $(k)$ in left panels of Fig. 2a, c, for the same two values of $\rho$.

In top panel, we observe that nodes in the active link network take values under the diagonal for large values of $\rho$. This comes from the definition of $G_{A L}$ and there is no different behavior for nodes in different states. 

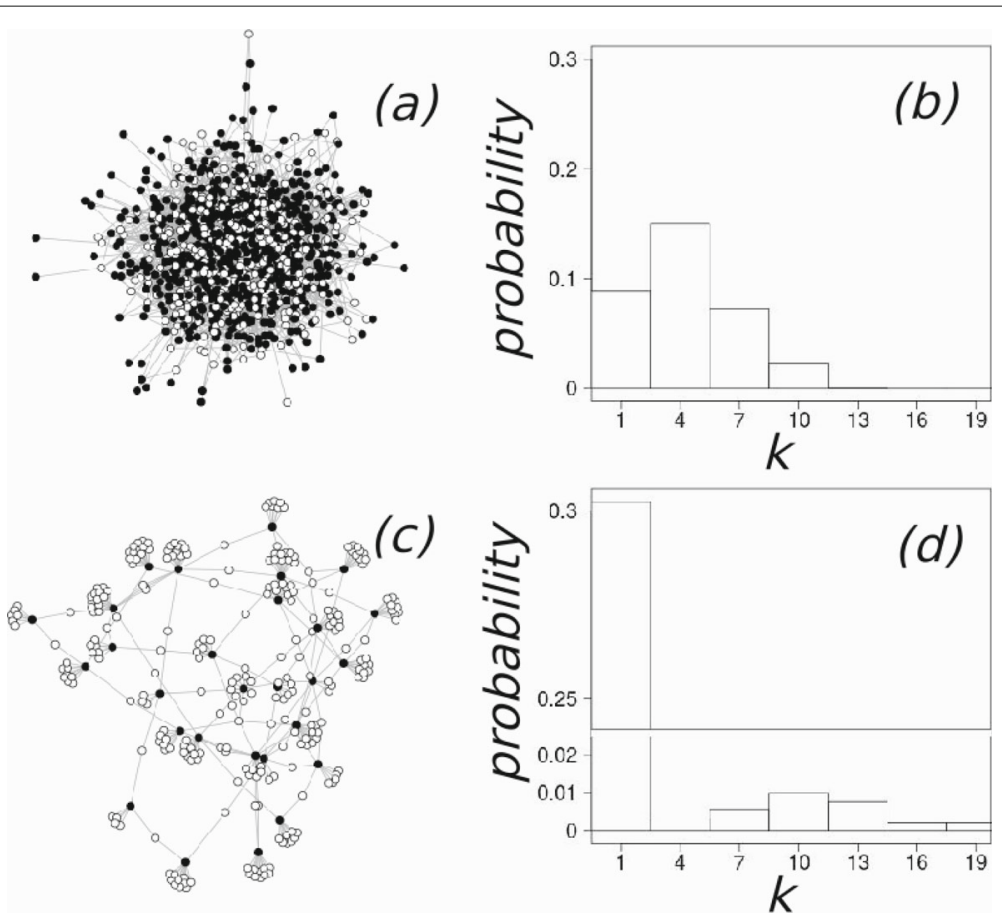

Fig. 1 Realization of a system with $N=1000$ agents, and Erdos-Renyi contact network of $<k>=12$. Figures correspond to two values of the parameter $\rho$. Up: early times $\rho=0.47$; Down: times close to convergence $\rho=0.026$. Left panel $\mathbf{a}, \mathbf{c}$ : snapshots of $G_{A L}$. Right panel $\mathbf{b}, \mathbf{d}$ : degree distribution of the corresponding graph $G_{A L}$
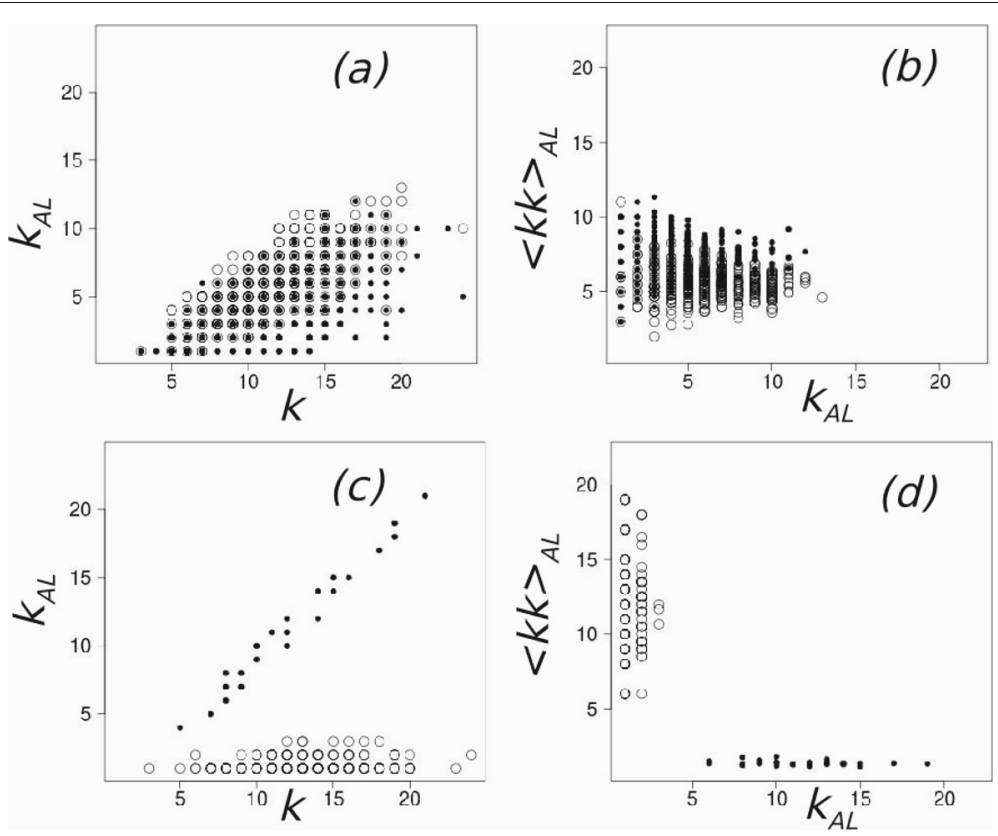

Fig. 2 Snapshots and degree distribution of $G_{A L}$ for Erdos-Renyi contact network of $<k>=12$ with $N=1000$ agents. Upper panels $\mathbf{a}$ and $\mathbf{b}$ correspond to $\rho=0.47$. Down panels $\mathbf{c}$ and $\mathbf{d}$ correspond to $\rho=0.026$. Left panels $\mathbf{a}$, $\mathbf{c}$ : degree of the nodes in $G_{A L}\left(k_{A L}\right)$ versus the degree of the nodes in the underlying static network (k). Right panels b, d: degree correlation of $G_{A L}$ for each agent of $G_{A L}$ 
When we look at the collective state at small values of $\rho$ we can observe that the dynamic leads the system to a state close to consensus and it is reflected in the connectivity properties of the nodes, consistently with the star-like motifs observed in the previous figure: few nodes with the approximately the same degree in the $G_{A L}$ and $G\left(k \simeq k_{A L}\right)$, and most of the nodes with $k_{A L}=1$ or $k_{A L}=2$, no matter their degrees in the ER static network.

In right panels of Fig. $2 \mathrm{~b}, \mathrm{~d}$ we plot the degree correlation of $G_{A L}$, i.e., the mean degree of the neighbors of the nodes of $G_{A L}\left(<k k>_{A L}\right)$ vs the degree of the same nodes in the Active Link Network $\left(k_{A L}\right)$. We observe a clear degree correlation among nodes of the $G_{A L}$ corresponding to the star-like motifs: neighbours of nodes with high degree have low mean degree and vice-versa. This is consistent with the large peak observed at low $k_{A L}$ and the small peak at intermediate values of $k_{A L}$ in the degree distribution of Fig. 1d.

From both figures we would like to point out that when the system is close to the absorbing state (values of $\rho$ close to zero), nodes are separated in accordance with their opinion state. In the present example, white circles correspond to small degree values while the mean degree of their neighbors is high. The opposite occurs for black circles. The snapshot of the corresponding $G_{A L}$ Fig. 1c reveals that white nodes are playing the role of leaves of stars, and black nodes the role of centres.

The main result can be summed up as follows: when the system converges, the topological role of the nodes splits according to their state in the network. The nodes that correspond to the dominant opinion display low degree in the Active Link Network and are connected to the nodes expressing the opposite opinion which have high degrees in star type motifs.

Given the different topological roles of both populations in the network $G_{A L}$, we found that it could be more informative to analyze its topological properties according to their dynamical states, i.e., separating populations by their opinions ( 1 or -1$)$.

Once we understand that it is informative to observe the properties of the network $G_{A L}$ for both populations in a separate way, we try to capture to what extent these two populations could be separate or not from the topological point of view of the network.

The results of Fig. 2 correspond to a single realization of the system, for two different values of the parameter $\rho$ that display the dynamics at different stages of the evolution. We can extrapolate these results to the case of several realizations of the system and, at the same time, for different values of $\rho$, thus trying to characterize the transition from large values of $\rho$ to small values.

In Fig. 3a, we plot the mean degree of the $G_{A L}\left(<k>_{A L}\right)$ as a function of $\rho$ for each one of the populations for 100 realizations of the system. For each time every 20 time steps, we averaged the values of degree for all agents in state $s=1$, and the same for agents in state $s=-1$. In this way, we obtain two values for each selected step. We can observe that, when the system evolves decreasing $\rho$, two branches separate from each other. The superior branch corresponds to the population which is playing the role of centres of starts, while the inferior branch is associated with the population that is playing the role of leaves of the stars. This separation is clear for low values of $\rho$. In order to clarify different stages of the evolution, we define colors for given ranges of $\rho$. In case we consider the two populations as a whole, both branches disappear and the mean degree of $G_{A L}$ decays from $k / 2$ when $\rho \simeq 0.5$ to a value near 2 while $\rho$ approaches to zero, as we can observe in Fig. 3b.

In order to understand the effects of the dynamics, we compare the Active Link Network $G_{A L}$ with a randomized version, $G_{A L}^{r}$. The way in which the random network is built (red 

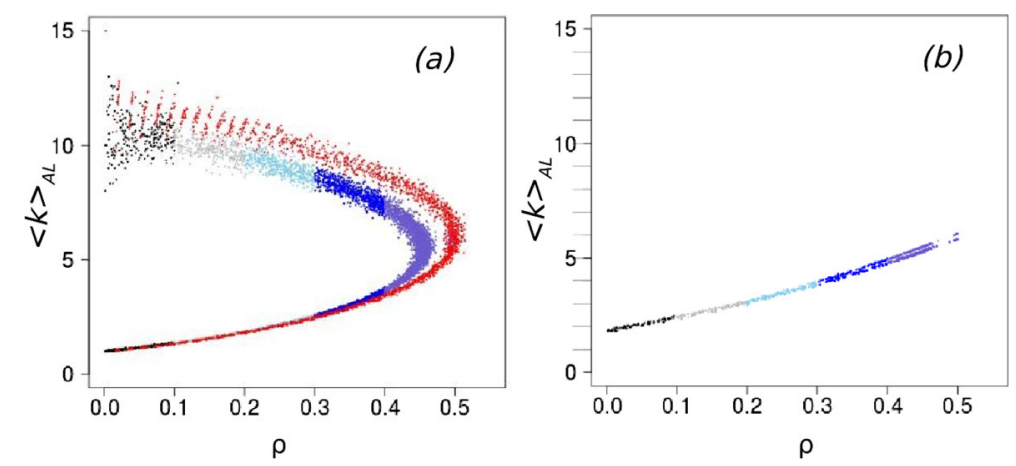

Fig. 3 Degree and degree correlations between the Erdos-Renyi contact network of $<k>=12$ and the $G_{A L}$ for $N=1000$ agents and 100 realizations of the dynamics. Panel $\mathbf{a}$ : mean degree of the $G_{A L}$ from each state's population vs $\rho$. Each step of realization contributes with two points to the plot: one for the mean degree of the population of agents in state 1 and one for agents in state -1 . Different colors are used to identify different ranges of values of $\rho$. Red points correspond to the case in which the states of the agents are assigned at random and the $G_{A L}^{r}$ is determined from this random assignation. Panel $\mathbf{b}$ : mean degree of the $G_{A L}$ vs $\rho$ without separating populations

points in Fig. 3a) consists in selecting the same number of agents with state $s=+1$ and $s=-1$ for a given time in the evolution and assigning them randomly to nodes. When comparing $G_{A L}$ with $G_{A L}^{r}$, the main difference is observed in the upper branch of the curve in Fig. 3a which is the minority of both populations. On the other hand, the bottom branch overlaps with $G_{A L}^{r}$, showing almost the same behavior when $\rho$ decreases. This if what we expect if a node of the majority population is placed at random in the underlying network: the chances to be alike as in the real Active Link Network are high.

Figure 4 shows the evolution of assortativity of the $G_{A L}\left(<k k>_{A L}\right.$ vs $k_{A L}$ ) for an ensemble of 100 realizations and the whole range of values of $\rho$, but separating nodes according to its opinion state as in Fig. 3. Once again, the case of low values of $\rho(<0.1)$ reflects a completely separated behavior of the populations (black points), while for large values of $\rho$ (violet points, corresponding to $\rho>0.4$ ), nodes with different opinions do not differentiate themselves. As in Fig. 3, red points correspond to random assignments of opinion state to nodes, without following the voter dynamics.

Once again we can observe that separating the populations of nodes by their dynamical state allows us to appreciate how different topological properties clearly emerge. In order to quantify the relation between the state of the nodes and their topological roles in the $G_{A L}$, we calculate their Normalized Mutual Information (NMI) according to the definition sketched in Appendix B.

Figure 5 shows $N M I$ as a function of $\rho$. We observe that, for high values of $\rho$, data about the state $s$ of the agents in the $G_{A L}$ does not bring information about their topological role thus $N M I$ is close to zero. On the other hand, when $\rho$ decreases, the fact of knowing one of the variables brings information about the other makes $N M I$ increases its value. The extreme scenario is reached when the Normalized Mutual Information has the maximum value 1: the knowledge of the state of nodes in $G_{A L}$ determines unequivocally their topological roles and vice-versa. These result are consistent with the observed in Figs. 1c, d and $2 \mathrm{c}, \mathrm{d}$.

As in Figs. 3 and 4, red points of Fig. 5 correspond to random assignment of opinion state to nodes, without following the voter dynamics. 


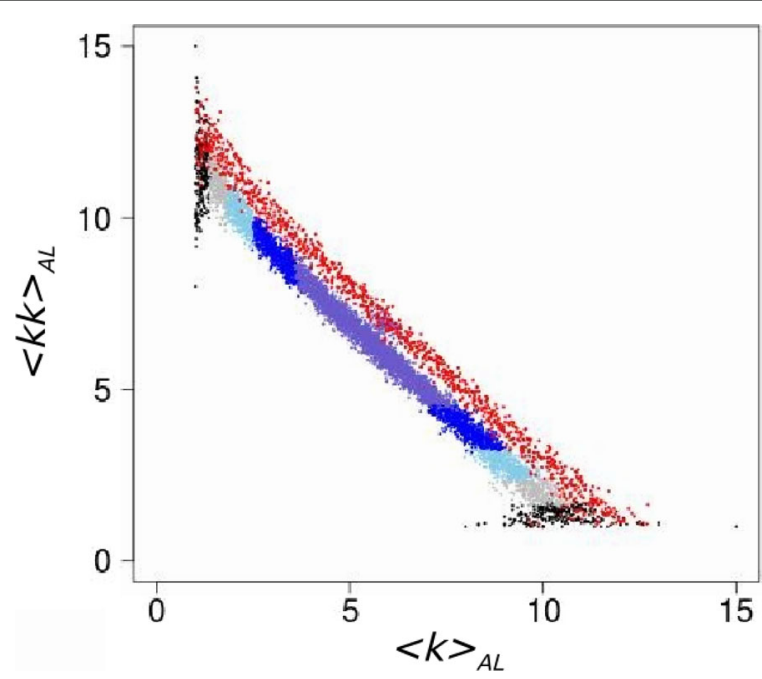

Fig. 4 Degree correlations of $G_{A L}$ for each agent of $G_{A L}$ separating nodes according to its opinion state for different values of $\rho .100$ realizations of $N=1000$ agents with an underlying network of contacts of Erdos-Renyi of $\langle k\rangle=12$. Each step of each realization contributes with two points to the plot: one for $<k k>_{A L}$ of the population of agents in state 1 and one for agents in state -1 . Different colors are used to identify different ranges of values of $\rho$. Red points correspond to the case in which the states of the agents are assigned at random and the $G_{A L}$ is determined from this random assignation

\section{Conclusions}

The voter model is a non-equilibrium dynamical model which has been widely studied from different points of view and with multiple variations. One of the most successful approaches used to describe the macroscopic dynamical evolution of the voter model is by describing the time evolution of the density of active links $\rho$, which goes from $\rho \simeq 0.5$ for a random initial condition to $\rho=0$ for the system near the absorbing state. This average magnitude has been used to compare analytical formulation of the model with numerical

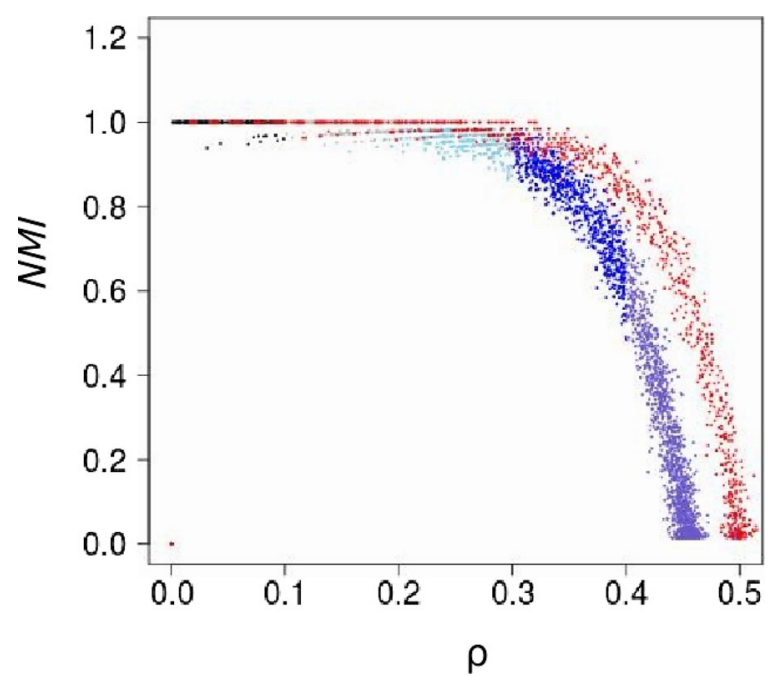

Fig. 5 Normalized Mutual Information (NMI) vs $\rho$ for 100 realizations of the system with $N=1000$ agents with an underlying network of contacts of Erdos-Renyi of $\langle k\rangle=12$. Red points correspond to the case in which the states of the agents are assigned at random and the $G_{A L}$ is determined from this random assignation 
calculations, as in (Vazquez and Eguiluz 2008), (Pugliese and Castellano 2009). However, the distribution of the active links over the contact network among nodes in different states serves as a map of the dynamical state of the system with much more information than simply its average value $\rho$.

In this paper we explore numerically the dynamics of active links in the voter model from a perspective of complex networks. This approach allows to comprehend how the dynamics of the model is mapped inside the topological features of a network of active links and how their dynamical evolution brings a more detailed understanding of the voter model.

At early times in the dynamics, the Active Links Network is similar to the underlying contact network, but when the system evolves towards the absorbing state, the graph of active links becomes highly dissasortative and a degree distribution dominates by a high peak at low degree values. When we look at the snapshots of Fig. 1, we can appreciate that it is dominated by star-like motifs and is completely different from the contact networks. These star-like structures are the signature that the voter model is closer to the consensus state.

The results displayed for the contact Erdos-Renyi network with mean degree $<k>=$ 12 are similar to those observed for the same kind of underlying network with different mean degree (ER with $<k>=6$ ), or for Barabasi-Albert scale free distribution, as is shown in the Appendix A.

An interesting result is that the description of Active Link Network is even more informative from topological point of view if nodes with state $s=+1$ and $s=-1$ are plotted separately. When the system is close to the consensus state, nodes from the majority population are the leaves of the star-like structures and therefore, they have degrees one or two in the active link network. If we plot the mean degree in the $G_{A L}$ or the mean degree of the nearest neighbors, we can observe how populations separate in different states when the systems evolves toward consensus (as $\rho$ decreases). Finally, Fig. 5 show that for $\rho \leq 0.3$ the state of the nodes determines their topological role in structures of the active link network, as indicated by the normalized mutual information.

This analytical framework could be used to analyze models with a more complex dynamics, like for instance the q-voter model (Timpanaro and Galam 2014; Javarone and Squartini 2015), the multistate voter model (Böhme and Gross 2012) or the Axelrod model (Axelrod 1997) where each node can see different number of networks and can be in different states respectively. In these cases, we do not expect that active link networks evolves towards star-like motifs of two states, because these structures are produced with two-states nodes only, like the classical voter model. However, these and other models deserve a careful further analysis.

Finally, we would like to point out that the use of complex networks for mapping the dynamical state of a complex system has been used in other fields to represent collective states when the underlying topology was unknown, as for instance in large scale brain experiments as in (Eguíluz et al. 2005), (Tsonis and Roebber 2004), but not as a tool to describe the evolution of a system towards asymptotic state, as was shown in this work.

\section{Appendix A}

In this Appendix we present the results when the voter model runs over different underlying contact networks. Figures A.1 and A.2 show the same results as Figs. 1 and 2 for scale-free 

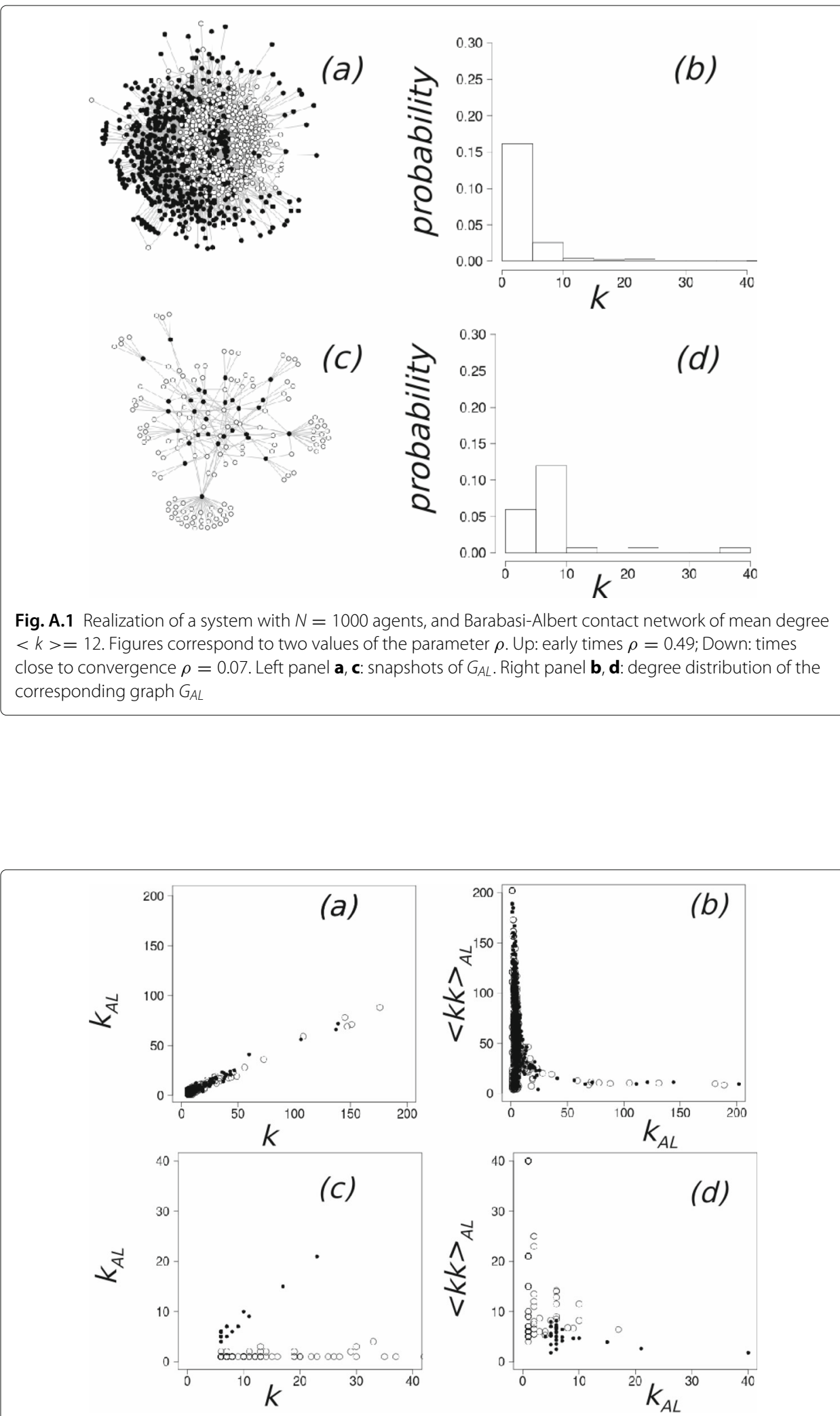

Fig. A.2 Realization of a system with $N=1000$ agents, and Barabasi-Albert contact network of $<k>=12$. Left panels $\mathbf{a}$, $\mathbf{c}$ : Degree of the nodes in $G_{A L}\left(k_{A L}\right)$ versus the degree of the nodes in the underlying static network (k). Right panels $\mathbf{b}$, d: degree correlation of $G_{A L}$ for each agent of $G_{A L}$. Upper panels $\mathbf{a}$ and $\mathbf{b}$ correspond to $\rho=0.49$. Lower panels $\mathbf{c}$ and $\mathbf{d}$ correspond to $\rho=0.07$ 


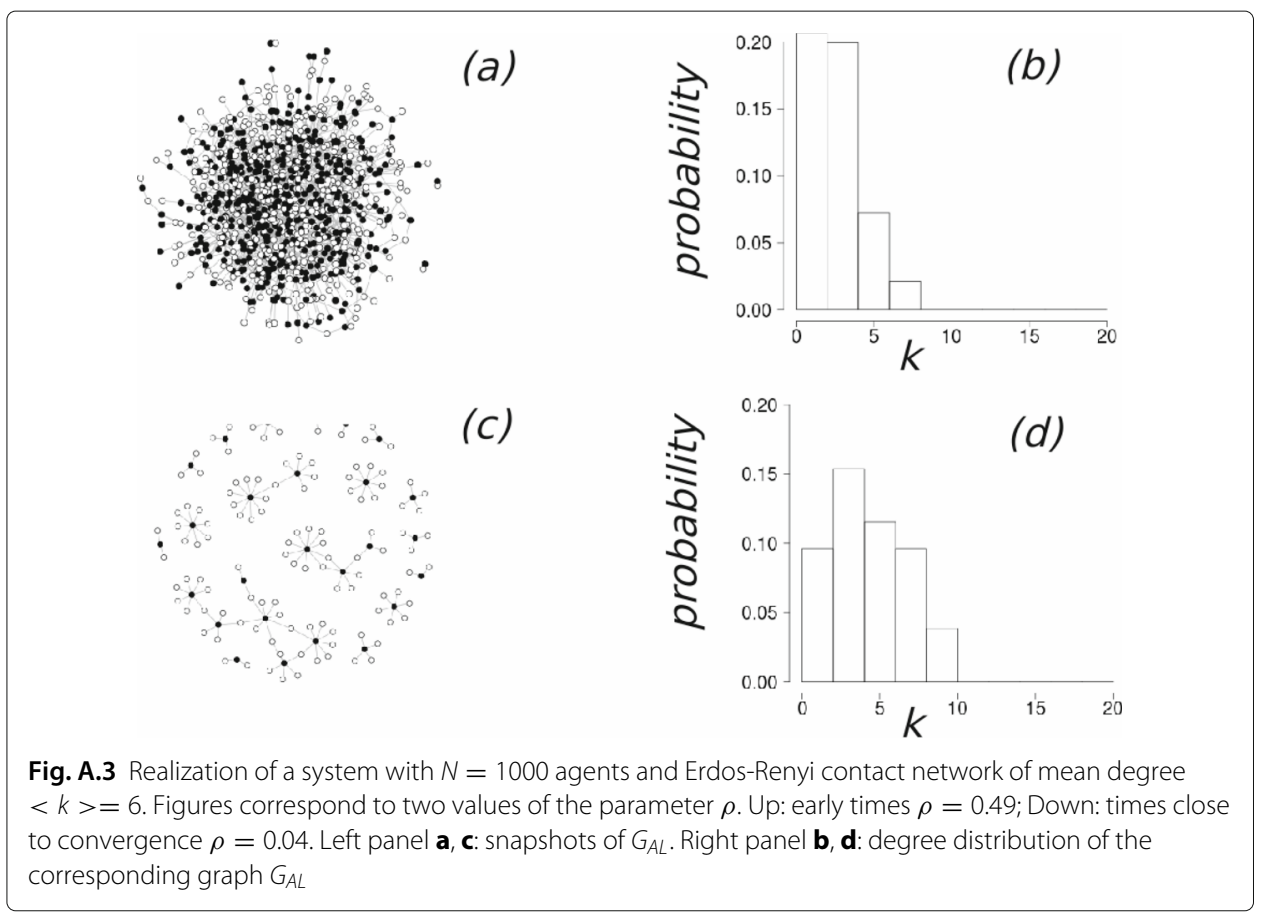

Barabasi-Albert $(<k>=12)$ contact networks. Upper panels correspond to initial stage of the evolution $(\rho=0.49)$, while bottom panels correspond to the almost converged system $(\rho=0.07)$. On the other hand, Figures A.3 and A.4 show the same result for Erdos-Renyi $<k>=6$ contact network. Upper panels correspond to $\rho=0.49$ value and bottom panels to $\rho=0.04$ value.
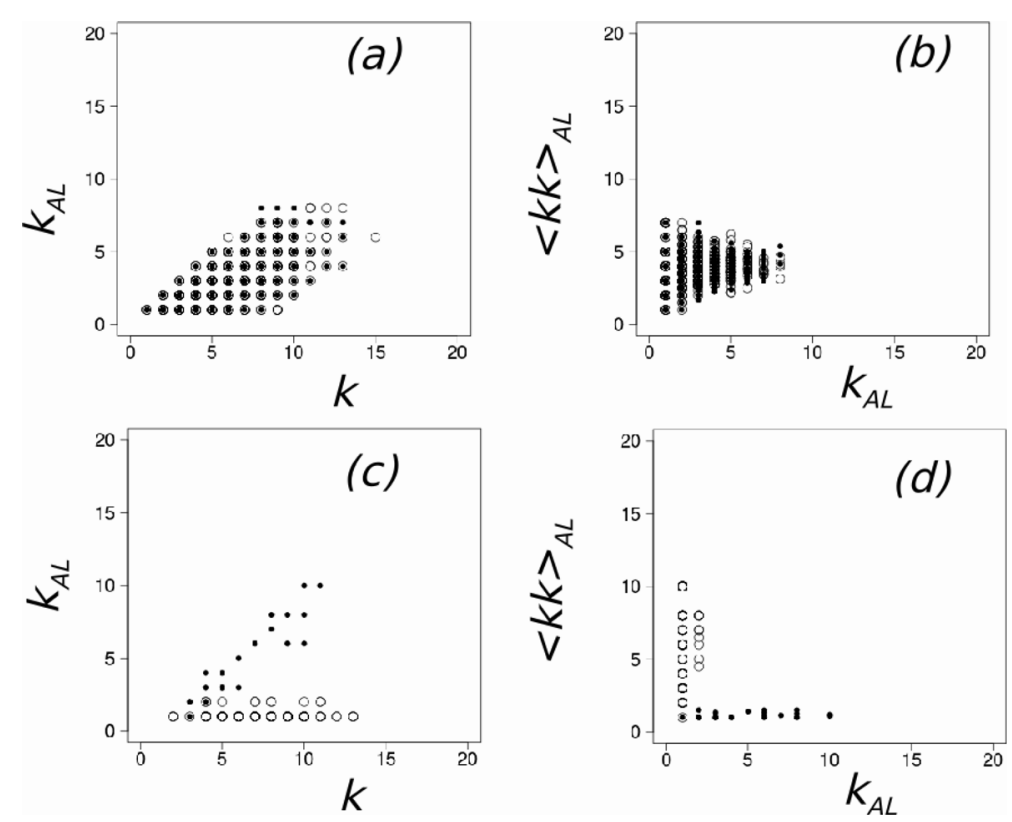

Fig. A.4 Realization of a system with $N=1000$ agents, and Erdos-Renyi contact network of mean degree $<k>=6$. Left panels $\mathbf{a}$, $\mathbf{c}$ : degree of the nodes in $G_{A L}\left(k_{A L}\right)$ versus the degree of the nodes in the underlying static network ( $k$ ). Right panels $\mathbf{b}$, d: degree correlation of $G_{A L}$ for each agent of $G_{A L}$. Upper panels $\mathbf{a}$ and $\mathbf{b}$ correspond to $\rho=0.49$. Lower panels $\mathbf{c}$ and $\mathbf{d}$ correspond to $\rho=0.04$ 
We can appreciate that the main conclusions about the behavior of the Active Link Networks for the Erdos-Renyi $\langle k>=12$ remain qualitatively the same for these two different contact networks: the system evolves towards $G_{A L}$ dominated by star-like motifs where nodes in different states play different topological roles. In the case of Erdos-Renyi, the differences between $G_{A L}$ and the contact networks are more evident than in the case of Barabasi-Albert. These differences are better appreciated when the mean degree of the network is increased. For the Barabasi-Albert case, although it is clear for the snapshot of the network (Figure A.2(c)) that one of the populations is playing the role of leaves and the other one the centres of stars, the difference between both networks $\left(G_{A L}\right.$ and the contact networks) is appreciated better in the relation between degrees than in the assortativity of $G_{A L}$.

\section{Appendix B}

In this appendix we provide the definition of Mutual Information used in Fig. 5. The mutual information of two variables $x$ and $y$ is defined as (Cover and Thomas 2006):

$$
M I(x, y)=H(x)-H(x \mid y)
$$

where $H(x \mid y)$ is the conditional entropy of variable $x$ given that $y$ is a known variable and $H(x)$ is the entropy of variable $x$. The entropy of a given variable $x$ is defined as the average of the Shannon Information contained in the possible outcomes of the variables, such as:

$$
H(x)=P(x=1) h(x=1)+P(x=0) h(x=0)
$$

being $P(x=1)$ the probability of variable $x$ taking value 1 and $h(x=1)$ the Shannon Information contained in the outcome value 1 of variable $x$, which is defined as $h(x=$ $1)=-\log _{2} P(x=1)$.

With the purpose of studying the relation between the state of the nodes and their topological roles, we define two categorical variables: the activity of an agent $a_{i}$, which takes the value 1 when belongs to the $G_{A L}$, and 0 otherwise, and the role of an agent $i, r_{i}$, which takes three possible values: $r_{i}=1$ if $k k_{A L}>k_{A L}$ (i agent is considered a leaf), $r_{i}=0$ if $k k_{A L}<k_{A L}$ ( $i$ agent is a centre of a star), and $r_{i}=2$ if $k k_{A L}=k_{A L}$ (otherwise).

The Normalized Mutual Information (NMI) is defined by $M I(r, s \mid a=1) / H(r \mid a=1)$, being $H(r \mid a=1)$ the Entropy of $r$, given that the nodes belong to the $G_{A L}$, and thus, the maximum value that $M I(r, s \mid a=1)$ can take.

Therefore, if the opinion states of the nodes determine unambiguously their topological roles in the $G_{A L}$, the Mutual Information NMI should be maximum. In the opposite case, when states of the nodes do not carry any information about the topological role, it should be zero.

\section{Acknowledgements}

We wold thank Lic. Ariel Salgado for enrichment discussions about the work.

\section{Authors' contributions}

$\mathrm{Cl}$ and $\mathrm{BP}$ designed the work; $\mathrm{Cl}$ and MS implemented the simulations; $\mathrm{Cl}$ prepared the figures; $\mathrm{Cl}, \mathrm{SV}$ and $\mathrm{BP}$ discussed the results and wrote the manuscript. All authors read and approved the final manuscript.

\section{Funding}

Conicet funding PIP 11220130100484 CO01.

\section{Availability of data and materials}

The datasets used and/or analysed during the current study are available from the corresponding author on reasonable request. 


\section{Competing interests}

The authors declare that they have no competing interests.

\section{Author details}

${ }^{1}$ Calculus Institute, University of Buenos Aires and CONICET, Buenos Aires, Argentina. ${ }^{2}$ Departamento de Física, Facultad de Ciencias Exactas y Naturales, Universidad de Buenos Aires, Buenos Aires, Argentina. ${ }^{3}$ Universidad de Buenos Aires. Facultad de Ciencias Económicas, Buenos Aires, Argentina. ${ }^{4}$ CONICET-Universidad de Buenos Aires. Instituto Interdisciplinario de Economía Política de Buenos Aires, Buenos Aires, Argentina. ${ }^{5}$ Instituto de Física de Buenos Aires (IFIBA), CONICET, Buenos Aires, Argentina.

Received: 9 July 2019 Accepted: 13 November 2019

Published online: 18 December 2019

\section{References}

Axelrod R (1997) The dissemination of culture a model with local convergence and global polarization. J Confl Resol 41(2):203-226

Bikhchandani S, Hirshleifer D, Welch I (1992) A Theory of Fads, Fashion, Custom, and Cultural-Change as Informational Cascades. J Pol Econ 100:992-1026

Böhme GE, Gross T (2011) Analytical calculation of fragmentation transitions in adaptive networks. Phys Rev E 83:035101 Böhme GA, Gross T (2012) Fragmentation transitions in multistate voter models. Phys Rev E 85:066117. https://doi.org/10. 1103/PhysRevE.85.066117

Castallano C, Fortunato S, Loreto V (2009) Statistical Physics of Social Dynamics. Rev Mod Phys 81:591

Castellano C (2005) Effect of network topology on the ordering dynamics of voter models. AIP Conf Proc 779:114

Castellano C, Vilone D, Vespignani A (2003) Incomplete ordering of the voter model on small-world networks. Europhys Lett 63:153-158

Clifford P, Sudbury A (1973) A model for spatial conflict. Biometrika 60:581-588

Cover TM, Thomas JA (2006) Elements of Information Theory. Wiley Series in Telecommunications and Signal Processing, United States of America

Cox J (1989) Coalescing Random Walks and Voter Model Consensus Times on the Torus in Zd.Ann. Probab 17:1333

Cox J, Griffeath D (1986) Diffusive Clustering in the Two Dimensional Voter Model. Ann Probab 14:347

Demirel G, Vazquez F, Böhme GE, Gross T (2014) Moment closure approximation for discrete adaptive networks. Phys D 67(267):68-80

de Oliveira, M, Mendes J, Santos M (1993) Nonequilibrium spin models with Ising universal behaviour. J Phys A 26:2317

Durrett R, et al. (2012) Graph fission in an evolving voter model. Proc Natl Acad Sci USA 109:3682

Eguíluz VM, Chialvo DR, Cecchi GA, Baliki M, Apkarian AV (2005) Scale-Free Brain Functional Networks. Phys Rev Lett 94:018102

Frachebourg L, Krapivsky PL (1996) Exact results for kinetics of catalytic reactions. Phys Rev E 53:R3009

Granovsky B, Madras N (1995) The noisy voter model. Stoch Proc Appl 55:23

Holley R, Liggett T (1975) Ergodic Theorems for Weakly Interacting Infinite Systems and the Voter Model. Ann Probab 3:643

Holme P, Newman MEJ (2006) Nonequilibrium phase transition in the coevolution of networks and opinions. Phys Rev E 74:056108

Javarone M, Squartini T (2015) Conformism-driven phases of opinion formation on heterogeneous networks: The q-voter model case. J Stat Mech Theory Exp:P10002. https://doi.org/10.1088/1742-5468/2015/10/P10002

Krapivsky PL (1992) Kinetics of a monomer-monomer model of heterogeneous catalysis. Phys Rev A 45:1067

Kimura D, Hayakawa Y (2008) Coevolutionary networks with homophily and heterophily. Phys Rev E 78:016103

Liggett TM (1999) Stochastic Interacting Systems: Contact, Voter and Exclusion Processes. Springer, New York

Liggett TM (1985) Interacting Particle Systems. Springer-Verlag, New York

Mobilia M (2003) Does a single zealot affect an infinite group of voters? Phys Rev Lett 91:028701

Mobilia M, Georgiev, IT (2005) Voting and catalytic processes with inhomogeneities. Phys Rev E 71:046102

Mobilia M, Petersen A, Redner S (2007) On the role of zealotry in the voter model. J Stat Mech: Theory Exp:P08029. https:// doi.org/10.1088/1742-5468/2007/08/p08029

Pugliese E, Castellano C (2009) Heterogeneous pair approximation for voter models on networks. EPL 88:58004

Redner S (2001) A Guide to First-passage Processes. Cambridge University Press, Cambridge

Scheucher M, Spohn H (1988) A soluble kinetic model for spinodal decomposition. J Stat Phys 53:279

Sood V, Antal T, Redner S (2008) Voter models on heterogeneous networks. Phys Rev E 77:041121

Sood V, Redner S (2005) Voter Model on Heterogeneous Graphs. Phys Rev Lett 94:178701

Suchecki K, Eguiluz V, San Miguel M (2005) Voter model dynamics in complex networks: Role of dimensionality, disorder, and degree distribution. Phys Rev E 72:036132

Suchecki K, Eguíluz VM, San Miguel M (2005) Conservation laws for the voter model in complex networks. Europhys Lett 69:228

Timpanaro AM, Galam S (2014) An analytical expres- sion for the exit probability of the q-voter model in one dimension. arxiv:1408.2734

Tsonis AA, Roebber PJ (2004) The architecture of the climate network. Phys A Stat Mech Appl 333:497-504

Vazquez F, Eguiluz VM (2008) Analytical solution of the voter model on uncorrelated networks. New J Phys 10:063011

Vazquez F, Eguíluz VM, San Miguel M (2008) Generic absorbing transition in coevolution dynamics. Phys Rev Lett 100:108702

Vázquez F, Krapivsky PL, Redner S (2003) Constrained opinion dynamics: freezing and slow evolution. J Phys A 36:L61

Vázquez F, Redner S (2004) Ultimate fate of constrained voters. J Phys A 37:8479

\section{Publisher's Note}

Springer Nature remains neutral with regard to jurisdictional claims in published maps and institutional affiliations. 